\title{
IMPACT OF INTERNATIONAL FINANCIAL REPORTING STANDARDS ON ACCOUNTING PRACTICES HARMONIZATION WITHIN EUROPEAN UNION -PARTICULAR CASE OF INTANGIBLE ASSETS-
}

\author{
Adriana Tiron Tudor ${ }^{l}$ \\ Ioana-Maria Dragu ${ }^{2}$
}

\begin{abstract}
This paper is meant to study the impact of IFRS on accounting practices harmonization, by measuring the degree in which different companies from Europe use same methods when reporting their intangible assets. Therefore, the objective of the research is to demonstrate the existence of harmonizing tendencies between European Union member states, as a result of IFRS adoption. The methodology implies Herfindahl Index computation for a sample of 51 listed companies that develop their activity in five European countries. The results suggest the influence of International Financial Reporting Standards on accounting practices, as there is evidence of high harmonization level for intangible assets. Many of the analysed situations recorded if not maximum harmonizing values at least visible tendencies to harmonize accounting practices.
\end{abstract}

Key words: accounting, harmonization, intangible, measurement.

JEL Code: $M 41$

\section{Introduction}

Ever since the Norwalk Agreement has been signed, FASB and IASB continue to collaborate in obtaining a single set of accounting regulations, which can serve for practical accounting purposes. As globalization implies the existence of unique financial reporting standards, one can state the importance of harmonizing international accounting regulations.

The aim of this paper is to present the impact of IFRS on accounting practices, by measuring the material harmonization degree for a sample of 51 listed companies that develop their activity in five European countries: Great Britain, Germany, Austria, Italy and France. The hypothesis of this study states that IFRS has generated accounting practices harmonization within European Union member states. In order to verify whether this statement is true or not, 51 companies were chosen, each of them corresponding to one of the mentioned states, and whose annual reports for 2009 have been subject to a detailed analysis. In particular, this paper investigates international harmonization for intangible assets. According to IAS 38, the main criteria for intangibility, of identification, nonmonetary and non-physical substance forms, are fulfilled by set up and development costs, goodwill, trademarks and brands, patents and licenses, customers lists. In addition, the study provides an analysis of how European companies report their intangibles, during the phases of an asset life of recognition, valuation and impairment.

The relevance of this research consists mainly in its contribution to international accounting, by establishing the harmonization level in the European Union concerning accounting practices, for the particular case of intangible assets. Therefore, measuring material harmonization for companies

\footnotetext{
",Babes-Bolyai” University of Cluj-Napoca, adriana.tiron@ ecom.ubbcluj.ro

${ }^{2}$ „Babes-Bolyai” University of Cluj-Napoca, ioanadragu@yahoo.com.
} 
activating in Europe, assumes in fact performing an analysis on an aggregate of accounting policies, which in turn concurs to the development of accounting field.

\section{Literature Review}

Emerging the globalisation process, it seems accounting standards harmonization encounters an expansion in the whole world, reducing divergence between different states' regulations (Reinstein and Weirich, 2002). In this context, international harmonization is given considerable attention, as it would lead to simplified procedures regarding financial reporting, so that those who develop the mentioned documents, as well as their users, will not have to comply with more types of accounting standards and laws.

In an attempt to study accounting harmonization in the public sector, some authors (Fuertes, 2008) define the harmonizing process, as being opposite to diversity and variation, or by expressing a decrease in contradictory rules and thus resulting in obtaining a higher comparability degree for financial reporting. Other scientists (Canibano and Mora, 2000) have chosen to present harmonization in contrast with standardization, by mentioning its realistic nature and trend towards a state of harmony. The study conducted by Chand and White (Chand and White, 2007: 606), reveals the differences between harmonization and convergence, the second finalizing with IFRS adoption. According to them, the path to an 'accountable world' is reached through harmonization or convergence, as a premise for a unique set of financial reports.

Accounting literature identifies a need for international harmonization in this field (Ramcharran, 2000), emerging especially from practice, where diversification of countries' regulations may interfere with the way financial information is perceived by its users (Combarros, 2000). However, according to various researchers (Ding et al., 2007), harmonizing accounting practices should not be seen as main purpose of organizations regulating this filed, as it influences a countries' national identity in terms of accounting regulations.

This paper has been elaborated after studying similar research on accounting practices (Capalbo, 2003) that approached comparative analysis in international accounting area. Furthermore, there were scientists that found evidence of accounting harmonization (Lin and Wang, 2001) when examining companies' financial reports. One representative study (Cazavan and Stolowy, 2001) has involved an attempt to determine if there is sign of accounting practices harmonizing tendencies with respect to intangible assets, on a global scale. The findings suggested that when considering elements from beyond European boundaries, intangibles' treatments applied by different states from all over the world stand for disharmony, which means global harmonization could be still far away from being implemented. In addition, research has been made regarding harmonization measurement in Europe (Canibano and Mora, 2000), analysing financial reports of European companies and using the $\mathrm{C}$ Index to express the harmonizing degree for certain accounting elements. The results showed there is not enough evidence to demonstrate harmonization tendencies for the chosen issues. Another study which implies annual reports' analysis (Hancock et al., 2002), revealed that accounting methods established by law or accounting profession under the form of regulations, do not necessarily interfere with the ones used by companies in practice. A distinct approach to international accounting harmonization could be to examine its influence on a certain country. Studies in this field (Kikuya, 2001) revealed countries willingness to harmonize that is not, however, materialised in practice, as differences still exist in the form of limitation for the harmonization process.

When measuring accounting practices harmonization, one tries in fact to determine the degree in which firms apply for same accounting methods or treatments. International accounting literature confers evidence of such measurement, by computing Herfindahl $\mathrm{H}$ Index and C Index as part of a statistical approach (Taplin, 2003). Another index used for harmonization disclosure purposes is the I Index, which generally follows a Chi-square statistics approach (Muhammad, 2006). With the help of this indicator, scientists can find prove of harmonization levels for practical 
accounting purposes. The three previously mentioned indicators, namely Herfindahl $\mathrm{H}$ Index, $\mathrm{C}$ Index and I Index, are considered to bring a significant contribution to the measurement international accounting harmony (Taplin, 2004).

\section{Research Methodology}

The research applied in this paper is based on a sample of 51 listed companies from five European Union member states, studying the impact of IFRS on accounting practices harmonization. In an attempt to determine material harmonization, we use the option concentration analysis, and this means computing the Herfindahl Index in order to obtain a synthesis of the harmonization degree at practical accounting level. In particular, the study assumes measuring the degree in which different companies from Europe use same methods when reporting their intangible assets. Regarding intangibles, these are analysed in accordance with three main stages, namely recognition, valuation and impairment. Having the objective to bring evidence for the existence of harmonizing tendencies between European Union member states, the research emerges from statistics analysis, as it examines the comparability for companies' practices from a European perspective.

\section{Analysis on option concentration}

The first step of the performed analysis involved data gathering. Therefore, the annual reports ${ }^{3}$ of the 51 companies implied in the research were consulted in order to find information regarding accounting policies used in case of intangible assets.

The sample firms were chosen as follows: ten from Great Britain, eleven from Germany, ten from Austria, nine from Italy and eleven from France (table no.1).

\section{Analysed Companies}

\begin{tabular}{|c|c|c|c|c|}
\hline $\begin{array}{l}\text { Great } \\
\text { Britain }\end{array}$ & Germany & Austria & Italy & France \\
\hline A.B. Foods & Travel24.com & Schoeller Bleckmann & Autogrill & Capmegini \\
\hline Cadbury & Commerzbank & Zumtobel AG & Mediobanca & CNP Assurances \\
\hline $\begin{array}{l}\text { Unilever } \\
\text { INTL }\end{array}$ & Curanum AG & Erste Group & Parmalat & Auchan \\
\hline $\begin{array}{l}\text { POWER } \\
\text { Scot\&STH }\end{array}$ & Bowe Systec & $\begin{array}{l}\text { Wienerberger AG } \\
\text { Flughhafen Wien }\end{array}$ & Pininfarina Group & Alcatel \\
\hline Energy & Wacker Chemie & GROUP & FIAT & Sanofi Aventis \\
\hline ICAP & Bayer & Strabag AG & Intessa San Paolo & Technicolor \\
\hline Man Group & Hexion & Viena Insurance Group & Luxotica & Total \\
\hline $\begin{array}{l}\text { Rexam } \\
\text { Smith }\end{array}$ & Axitron & OMV & ENI & Veolia \\
\hline Group & GO YELLOW & Telecom & ACTA & Atari \\
\hline \multirow[t]{2}{*}{ Inmarsat } & $4 \mathrm{SC}$ & Intercell & & Parrot \\
\hline & Arial Bank & & & EADS \\
\hline
\end{tabular}

Measurement of material harmonization for the sample of 51 firms implies Herfindahl $\mathrm{H}$ Index computation. In addition, the following formula is used (Taplin, 2003: 83):

$$
H=\sum p_{i}^{2}
$$

\footnotetext{
${ }^{3}$ All the implied documents in the form of annual reports of the 51 analyzed companies represent data for year 2009.
} 
According to Taplin (Taplin, 2003), $\mathrm{H}$ represents Herfindahl Index, $\mathrm{p}_{\mathrm{i}}$ is the relative frequency in correlation with the accounting method $\mathrm{i}$, and finally $\mathrm{i}$ takes values from 1 to $\mathrm{M}$, where $M$ consists in the number of accounting methods.

$\mathrm{H}$ Index analysis has been performed for each of the main stages of intangible assets, determining the number of used accounting treatments, respectively the ones that are not used or not mentioned by accounting policies from companies' annual reports. In the first case, we computed $\mathrm{H}$ Index for the elements recognised as intangible assets (table no.2). However, before computing this index we have identified the corresponding accounting options or treatments, established how many companies have chosen the method, and calculated the absolute frequency and the relative one. Absolute frequency represents the percentage of companies that use or do not use or mention the respective treatment, while relative frequency is the decimal number corresponding to the percentage. For instance, concerning goodwill, it seems that all the selected companies recognise it as an intangible asset, resulting in the fact that $\mathrm{H}$ Index becomes 1 and thus takes the maximum value, which in turn leads to the conclusion of a maximum harmonization degree for this element. On the other hand, in case of customers' lists, there are 36 firms that recognise it, while the rest of 15 entities do not mention or do not use them. For this element $\mathrm{H}$ Index becomes 0.6, which can be interpreted as medium harmonization degree.

Table no. 2

Elements recognised as intangible assets

\begin{tabular}{ccccc}
$\begin{array}{c}\text { Treatment/ Accounting } \\
\text { option }\end{array}$ & $\begin{array}{c}\text { Companies } \\
\text { No. }\end{array}$ & Frequency & pi & $\begin{array}{c}\text { H } \\
\text { Index }\end{array}$ \\
\hline RECOGNITION & 51 & $100 \%$ & 1 & \\
\hline Set up costs & 12 & $24 \%$ & 0,2353 & 0,664 \\
\hline a) Used & 39 & $76 \%$ & 0,7800 & \\
b) Not used/Not mentioned & 51 & $100 \%$ & 1 & \\
\hline Development costs & 39 & $76 \%$ & 0,7647 & 0,640 \\
\hline a) Used & 12 & $24 \%$ & 0,2353 & \\
b) Not used/Not mentioned & 51 & $100 \%$ & 1 & \\
\hline Goodwill & 51 & $100 \%$ & 1 & 1 \\
\hline a) Used & 0 & $0 \%$ & 0 & \\
b) Not used/Not mentioned & 51 & $100 \%$ & 1 & \\
\hline Trademarks/Brands & 50 & $98 \%$ & 0,980 & \multirow{2}{*}{0,962} \\
\hline a) Used & 1 & $2 \%$ & 0,020 & \\
b) Not used/Not mentioned & 51 & $100 \%$ & 1 & \\
\hline Patents/Licences & 45 & $88 \%$ & 0,8824 & \multirow{2}{*}{0,792} \\
\hline a) Used & 6 & $12 \%$ & 0,1176 & \\
\hline b) Not used/Not mentioned & 51 & $100 \%$ & 1 & \\
\hline Customers' Lists & 36 & $71 \%$ & 0,7200 & \multirow{2}{*}{0,608} \\
\hline a) Used & 15 & $29 \%$ & 0,3000 & \\
b) Not used/Not mentioned & & & & \\
\hline
\end{tabular}

Regarding the valuation of intangible assets, the study was decomposed into more parts, in correlation to the analysed element of intangibles. The first part comprises the valuation for set up costs (table no.3). The analysis was performed using the same methodology or steps presented at recognition of intangible assets, with the specification that it covers initial and revaluation methods for set up costs only. Although it seems that most of the analyse companies do not use or do not 
mention their valuation methods, there is a visible tendency for practices harmonization, the values for $\mathrm{H}$ Index exceeding 0.8 and even achieving 1, that stands for the maximum point of the harmonizing process.

Table no. 3

\section{Valuation of Set up Costs}

\begin{tabular}{lcccc}
$\begin{array}{c}\text { Treatment/ } \\
\text { Accounting option }\end{array}$ & $\begin{array}{c}\text { Companies } \\
\text { No. }\end{array}$ & Frequency & pi & $\begin{array}{c}\text { H } \\
\text { Index }\end{array}$ \\
\hline VALUATION & & & & \\
\hline Initial Valuation & & & & \\
\hline Acquisition cost & 51 & $100 \%$ & 1 & \\
\hline a) Used & 4 & $8 \%$ & 0,0784 & 0,85544 \\
b) Not used/Not mentioned & 47 & $92 \%$ & 0,9216 & \\
\hline Production cost & 51 & $100 \%$ & 1 & \\
\hline a) Used & 4 & $8 \%$ & 0,0784 & 0,855 \\
b) Not used/Not mentioned & 47 & $92 \%$ & 0,9216 & \\
\hline Revaluation & & & & \\
\hline Book Value & 51 & $100 \%$ & 1 & \\
\hline a) Used & 5 & $10 \%$ & 0,0980 & 0,823 \\
b) Not used/Not mentioned & 46 & $90 \%$ & 0,9020 & \\
\hline Fair Value & 51 & $100 \%$ & 1 & \\
\hline a) Used & 0 & $0 \%$ & 0 & 1 \\
b) Not used/Not mentioned & 51 & $100 \%$ & 1 &
\end{tabular}

Concerning the valuation of development costs, with few exceptions, there is evidence of medium accounting practices harmonization (table no.4)

Table no. 4

Valuation of Development Costs

\begin{tabular}{lcccc}
$\begin{array}{c}\text { Treatment/ } \\
\text { Accounting option }\end{array}$ & $\begin{array}{c}\text { Companies } \\
\text { No. }\end{array}$ & Frequency & pi & $\begin{array}{c}\text { H } \\
\text { Index }\end{array}$ \\
\hline VALUATION & & & & \\
\hline Initial Valuation & & & & \\
\hline Acquisition cost & 51 & $100 \%$ & 1 & \\
\hline a) Used & 21 & $41 \%$ & 0,4118 & 0,51557 \\
b) Not used/Not mentioned & 30 & $59 \%$ & 0,5882 & \\
\hline Production cost & 51 & $100 \%$ & 1 & \\
\hline a) Used & 18 & $35 \%$ & 0,3529 & \multirow{2}{*}{0,543} \\
b) Not used/Not mentioned & 33 & $65 \%$ & 0,6471 & \\
\hline Revaluation & & \multicolumn{2}{c}{1} & \\
\hline Book Value & 51 & $100 \%$ & 0,3725 & \multirow{2}{*}{0,532} \\
\hline a) Used & 19 & $37 \%$ & $0,37 \%$ & \\
b) Not used/Not mentioned & 32 & $63 \%$ & 0,6275 & \\
\hline Fair Value & 51 & $100 \%$ & 1 & \\
\hline a) Used & 6 & $12 \%$ & 0,1176 & \multirow{2}{*}{0,792} \\
b) Not used/Not mentioned & 45 & $88 \%$ & 0,8824 &
\end{tabular}


Valuation of goodwill demonstrates, as in the previous case, a medium level for harmonization, excepting for production cost issue, method used by $98 \%$ of the analysed companies for which $\mathrm{H}$ Index indicates high level of harmonizing (table no.5).

Table no. 5

\begin{tabular}{lcccc}
\multicolumn{2}{c}{ Valuation of Goodwill } & & \\
$\begin{array}{c}\text { Treatment/ } \\
\text { Accounting option }\end{array}$ & $\begin{array}{c}\text { Companies } \\
\text { No. }\end{array}$ & Frequency & pi & $\begin{array}{c}\text { Hox } \\
\text { Index }\end{array}$ \\
\hline VALUATION & & & & \\
\hline Initial Valuation & & & & \\
\hline Acquisition cost & 51 & $100 \%$ & 1 & \\
\hline a) Used & 40 & $78 \%$ & 0,7843 & \multirow{2}{*}{0,661} \\
b) Not used/Not mentioned & 11 & $22 \%$ & 0,2157 & \\
\hline Production cost & 51 & $100 \%$ & 1 & \\
\hline a) Used & 1 & $2 \%$ & 0,0196 & \multirow{2}{*}{0,962} \\
b) Not used/Not mentioned & 50 & $98 \%$ & 0,9804 & \\
\hline Revaluation & & & & \\
\hline Book Value & 51 & $100 \%$ & 1 & \\
\hline a) Used & 24 & $47 \%$ & 0,4706 & \multirow{2}{*}{0,502} \\
b) Not used/Not mentioned & 27 & $53 \%$ & 0,5294 & \\
\hline Fair Value & 51 & $100 \%$ & 1 & \\
\hline a) Used & 22 & $43 \%$ & 0,4314 & \multirow{2}{*}{0,509} \\
b) Not used/Not mentioned & 29 & $57 \%$ & 0,5686 & \\
& & & &
\end{tabular}

In case of valuation of brands and trademarks, we can observe that $\mathrm{H}$ Index has medium values that lead to the conclusion of recording a medium level for accounting harmonization (table no.6).

Valuation of Brands and Trademarks

Table no. 6

\begin{tabular}{lcccc}
$\begin{array}{c}\text { Treatment/ } \\
\text { Accounting option }\end{array}$ & $\begin{array}{c}\text { Companies } \\
\text { No. }\end{array}$ & Frequency & pi & $\begin{array}{c}\text { H } \\
\text { Index }\end{array}$ \\
\hline VALUATION & & & & \\
\hline Initial Valuation & & & & \\
\hline Acquisition cost & 51 & $100 \%$ & 1 & \\
\hline a) Used & 28 & $55 \%$ & 0,5490 & \multirow{2}{*}{0,504} \\
b) Not used/Not mentioned & 23 & $45 \%$ & 0,4510 & \\
\hline Production cost & 51 & $100 \%$ & 1 & \\
\hline a) Used & 11 & $22 \%$ & 0,2157 & \multirow{2}{*}{0,662} \\
b) Not used/Not mentioned & 40 & $78 \%$ & 0,7843 & \\
\hline Revaluation & & & & \\
\hline Book Value & 51 & $100 \%$ & 1 & \\
\hline a) Used & 22 & $43 \%$ & 0,4314 & \multirow{2}{*}{0,509} \\
b) Not used/Not mentioned & 29 & $57 \%$ & 0,5686 & \\
\hline Fair Value & 51 & $100 \%$ & 1 & \\
\hline a) Used & 15 & $29 \%$ & 0,2941 & \multirow{2}{*}{0,585} \\
b) Not used/Not mentioned & 36 & $71 \%$ & 0,7059 &
\end{tabular}


Patents and licences valuation imply a medium level for harmonization, the highest value of $\mathrm{H}$ Index being recorded for fair value method or revaluation method, of only 0.64 (table no.7).

\section{Valuation of Patents and Licenses}

Table no. 7

\begin{tabular}{lcccc}
$\begin{array}{c}\text { Treatment/ } \\
\text { Accounting option }\end{array}$ & $\begin{array}{c}\text { Companies } \\
\text { No. }\end{array}$ & Frequency & pi & $\begin{array}{c}\text { H } \\
\text { Index }\end{array}$ \\
\hline VALUATION & & & & \\
\hline Initial Valuation & & & & \\
\hline Acquisition cost & 51 & $100 \%$ & 1 & \\
\hline a) Used & 30 & $59 \%$ & 0,5882 & 0,515 \\
b) Not used/Not mentioned & 21 & $41 \%$ & 0,4118 & \\
\hline Production cost & 51 & $100 \%$ & 1 & \\
\hline a) Used & 13 & $25 \%$ & 0,2549 & \multirow{2}{*}{0,620} \\
b) Not used/Not mentioned & 38 & $75 \%$ & 0,7451 & \\
\hline Revaluation & & & & \\
\hline Book Value & 51 & $100 \%$ & 1 & \\
\hline a) Used & 19 & $37 \%$ & 0,3725 & \multirow{2}{*}{0,532} \\
b) Not used/Not mentioned & 32 & $63 \%$ & 0,6275 & \\
\hline Fair Value & 51 & $100 \%$ & 1 & \\
\hline a) Used & 12 & $24 \%$ & 0,2353 & \multirow{2}{*}{0,640} \\
b) Not used/Not mentioned & 39 & $76 \%$ & 0,7647 &
\end{tabular}

In case of customers' lists, the findings reveal again a medium harmonization for valuation (table no.8).

\section{Valuation of Customer Lists}

Table no. 8

\begin{tabular}{lcccc}
$\begin{array}{c}\text { Treatment/ } \\
\text { Accounting option }\end{array}$ & $\begin{array}{c}\text { Companies } \\
\text { No. }\end{array}$ & Frequency & pi & $\begin{array}{c}\text { H } \\
\text { Index }\end{array}$ \\
\hline VALUATION & & & & \\
\hline Initial Valuation & & & & \\
\hline Acquisition cost & 51 & $100 \%$ & 1 & \\
\hline a) Used & 26 & $51 \%$ & 0,5098 & 0,500 \\
b) Not used/Not mentioned & 25 & $49 \%$ & 0,4902 & \\
\hline$\quad$ Production cost & 51 & $100 \%$ & 1 & \\
\hline a) Used & 12 & $24 \%$ & 0,2353 & \multirow{2}{*}{0,640} \\
b) Not used/Not mentioned & 39 & $76 \%$ & 0,7647 & \\
\hline Revaluation & & & & \\
\hline Book Value & 51 & $100 \%$ & 1 & \\
\hline a) Used & 17 & $33 \%$ & 0,3333 & \multirow{2}{*}{0,556} \\
b) Not used/Not mentioned & 34 & $67 \%$ & 0,6667 & \\
\hline Fair Value & 51 & $100 \%$ & 1 & \\
\hline a) Used & 13 & $25 \%$ & 0,2549 & \multirow{2}{*}{0,620} \\
b) Not used/Not mentioned & 38 & $75 \%$ & 0,7451 &
\end{tabular}


Another stage in an asset life is represented by impairment. Firstly, we analyse impairment for set up costs (table no.9). This analysis reveals that most of the companies do not mention or do not use impairment for their set up costs, and H Index registers high values, that exceed 0.7. As for harmonization, it reaches its maximum in case of digressive and accelerated methods of depreciation, while presenting a strong tendency to harmonize.

\section{Impairment for Set up Costs}

Table no. 9

\begin{tabular}{|c|c|c|c|c|}
\hline $\begin{array}{c}\text { Treatment/ } \\
\text { Accounting } \\
\text { option }\end{array}$ & $\begin{array}{c}\text { Companies } \\
\text { No. }\end{array}$ & Frequency & pi & $\begin{array}{c}\mathbf{H} \\
\text { Index }\end{array}$ \\
\hline \multicolumn{5}{|l|}{ IMPAIRMENT } \\
\hline \multicolumn{5}{|l|}{ Determination Models } \\
\hline Impariment testing & 51 & $100 \%$ & 1 & \\
\hline a) Used & 2 & $4 \%$ & 0,0392 & \multirow{2}{*}{0,925} \\
\hline b) Not used/Not mentioned & 49 & $96 \%$ & 0,9608 & \\
\hline Amortization & 51 & $100 \%$ & 1 & \\
\hline a) Used & 6 & $12 \%$ & 0,1176 & \multirow{2}{*}{0,792} \\
\hline b) Not used/Not mentioned & 45 & $88 \%$ & 0,8824 & \\
\hline \multicolumn{5}{|l|}{ Amortization Methods } \\
\hline Linear/Straight Line & 51 & $100 \%$ & 1 & \\
\hline a) Used & 5 & $10 \%$ & 0,0980 & \multirow{2}{*}{0,823} \\
\hline b) Not used/Not mentioned & 46 & $90 \%$ & 0,9020 & \\
\hline Digressive & 51 & $100 \%$ & 1 & \\
\hline a) Used & 0 & $0 \%$ & 0 & \multirow{2}{*}{1} \\
\hline b) Not used/Not mentioned & 51 & $100 \%$ & 1 & \\
\hline Accelerated & 51 & $100 \%$ & 1 & \\
\hline a) Used & 0 & $0 \%$ & 0 & \multirow{2}{*}{1} \\
\hline b) Not used/Not mentioned & 51 & $100 \%$ & 1 & \\
\hline Production Units & 51 & $100 \%$ & 1 & \\
\hline a) Used & 1 & $2 \%$ & 0,0196 & \multirow{2}{*}{0,962} \\
\hline b) Not used/Not mentioned & 50 & $98 \%$ & 0,9804 & \\
\hline
\end{tabular}

Impairment for development costs (table no.10) results in a medium harmonization degree in case of determination models and linear amortization. However, for the last three amortization methods, namely digressive, accelerated and production units, H Index indicates a high level for practices harmonization. 
Impairment for Development Costs

Table no. 10

\section{Treatment/}

Accounting option

\section{Companies Frequency pi H Index} No.

\begin{tabular}{|c|c|c|c|c|}
\hline IMPAIRMENT & & & & \\
\hline \multicolumn{5}{|l|}{ Determination Models } \\
\hline Impariment testing & 51 & $100 \%$ & 1 & \\
\hline a) Used & 10 & $20 \%$ & 0,1961 & \multirow{2}{*}{0,685} \\
\hline b) Not used/Not mentioned & 41 & $80 \%$ & 0,8039 & \\
\hline Amortization & 51 & $100 \%$ & 1 & \\
\hline a) Used & 35 & $69 \%$ & 0,6863 & \multirow{2}{*}{0,569} \\
\hline b) Not used/Not mentioned & 16 & $31 \%$ & 0,3137 & \\
\hline \multicolumn{5}{|l|}{ Amortization Methods } \\
\hline Linear/Straight Line & 51 & $100 \%$ & 1 & \\
\hline a) Used & 28 & $55 \%$ & 0,5490 & \multirow{2}{*}{0,505} \\
\hline b) Not used/Not mentioned & 23 & $45 \%$ & 0,4510 & \\
\hline Digressive & 51 & $100 \%$ & 1 & \\
\hline a) Used & 0 & $0 \%$ & 0 & \multirow{2}{*}{1} \\
\hline b) Not used/Not mentioned & 51 & $100 \%$ & 1 & \\
\hline Accelerated & 51 & $100 \%$ & 1 & \\
\hline a) Used & 2 & $4 \%$ & 0,0392 & \multirow{2}{*}{0,925} \\
\hline b) Not used/Not mentioned & 49 & $96 \%$ & 0,9608 & \\
\hline Production Units & 51 & $100 \%$ & 1 & \\
\hline a) Used & 2 & $4 \%$ & 0,0392 & \multirow{2}{*}{0,925} \\
\hline b) Not used/Not mentioned & 49 & $96 \%$ & 0,9608 & \\
\hline
\end{tabular}

As for goodwill impairment (table no.11), it suggests strong evidence of harmonization tendencies, with very high index values. 


\section{Impairment for Goodwill}

Table no. 11

\begin{tabular}{|c|c|c|c|c|}
\hline $\begin{array}{c}\text { Treatment/ } \\
\text { Accounting option }\end{array}$ & $\begin{array}{c}\text { Companies } \\
\text { No. }\end{array}$ & Frequency & pi & H Index \\
\hline \multicolumn{5}{|l|}{ IMPAIRMENT } \\
\hline \multicolumn{5}{|l|}{ Determination Models } \\
\hline Impariment testing & 51 & $100 \%$ & 1 & \\
\hline a) Used & 48 & $94 \%$ & 0,9412 & \multirow{2}{*}{0,889} \\
\hline b) Not used/Not mentioned & 3 & $6 \%$ & 0,0588 & \\
\hline Amortization & 51 & $100 \%$ & 1 & \multirow{3}{*}{0,925} \\
\hline a) Used & 2 & $4 \%$ & 0,0392 & \\
\hline b) Not used/Not mentioned & 49 & $96 \%$ & 0,9608 & \\
\hline \multicolumn{5}{|l|}{ Amortization Methods } \\
\hline Linear/Straight Line & 51 & $100 \%$ & 1 & \\
\hline a) Used & 1 & $2 \%$ & 0,0196 & \multirow{2}{*}{0,962} \\
\hline b) Not used/Not mentioned & 50 & $98 \%$ & 0,9804 & \\
\hline Degressive & 51 & $100 \%$ & 1 & \multirow{3}{*}{1} \\
\hline a) Used & 0 & $0 \%$ & 0 & \\
\hline b) Not used/Not mentioned & 51 & $100 \%$ & 1 & \\
\hline Accelerated & 51 & $100 \%$ & 1 & \multirow[b]{3}{*}{1} \\
\hline a) Used & 0 & $0 \%$ & 0 & \\
\hline b) Not used/Not mentioned & 51 & $100 \%$ & 1 & \\
\hline Production Units & 51 & $100 \%$ & 1 & \\
\hline a) Used & 1 & $2 \%$ & 0,0196 & \multirow{2}{*}{0,962} \\
\hline b) Not used/Not mentioned & 50 & $98 \%$ & 0,9804 & \\
\hline
\end{tabular}

Regarding brands and trademarks (table no.12), their impairment seems to result in medium to maximum harmonization, as determination models and linear amortization register $\mathrm{H}$ Index values around 0,5 while digressive, accelerated and production units methods imply high harmonizing tendencies. 


\section{Impairment for Brands and Trademarks}

Table no. 12

\section{Treatment/}

Accounting Companies Frequency pi H Index option No.

\begin{tabular}{|c|c|c|c|c|}
\hline IMPAIRMENT & & & & \\
\hline \multicolumn{5}{|l|}{ Determination Models } \\
\hline Impariment testing & 51 & $100 \%$ & 1 & \\
\hline a) Used & 18 & $35 \%$ & 0,3529 & \multirow{2}{*}{0,543} \\
\hline b) Not used/Not mentioned & 33 & $65 \%$ & 0,6471 & \\
\hline Amortization & 51 & $100 \%$ & 1 & \\
\hline a) Used & 25 & $49 \%$ & 0,4902 & \multirow{2}{*}{0,500} \\
\hline b) Not used/Not mentioned & 26 & $51 \%$ & 0,5098 & \\
\hline \multicolumn{5}{|l|}{ Amortization Methods } \\
\hline Linear/Straight Line & 51 & $100 \%$ & 1 & \\
\hline a) Used & 20 & $39 \%$ & 0,3922 & \multirow{2}{*}{0,523} \\
\hline b) Not used/Not mentioned & 31 & $61 \%$ & 0,6078 & \\
\hline Digressive & 51 & $100 \%$ & 1 & \\
\hline a) Used & 0 & $0 \%$ & 0 & \multirow{2}{*}{1} \\
\hline b) Not used/Not mentioned & 51 & $100 \%$ & 1 & \\
\hline Accelerated & 51 & $100 \%$ & 1 & \\
\hline a) Used & 1 & $2 \%$ & 0,0196 & \multirow{2}{*}{0,962} \\
\hline b) Not used/Not mentioned & 50 & $98 \%$ & 0,9804 & \\
\hline Production Units & 51 & $100 \%$ & 1 & \\
\hline a) Used & 0 & $0 \%$ & 0 & \multirow[t]{2}{*}{1} \\
\hline b) Not used/Not mentioned & 51 & $100 \%$ & 1 & \\
\hline
\end{tabular}

Concerning impairment for patents and licenses (table no.13), it appears that most of $\mathrm{H}$ Index value are over 0,6 indicating in general a high degree of harmonization. 
Impairment for Patents and Licenses

Treatment/

Accounting Companies Frequency pi H Index option No.

Table no. 13

\begin{tabular}{|c|c|c|c|c|}
\hline IMPAIRMENT & & & & \\
\hline \multicolumn{5}{|l|}{ Determination Models } \\
\hline Impariment testing & 51 & $100 \%$ & 1 & \\
\hline a) Used & 6 & $12 \%$ & 0,1176 & \multirow{2}{*}{0,792} \\
\hline b) Not used/Not mentioned & 45 & $88 \%$ & 0,8824 & \\
\hline Amortization & 51 & $100 \%$ & 1 & \\
\hline a) Used & 37 & $73 \%$ & 0,7255 & \multirow{2}{*}{0,602} \\
\hline b) Not used/Not mentioned & 14 & $27 \%$ & 0,2745 & \\
\hline \multicolumn{5}{|l|}{ Amortization Methods } \\
\hline Linear/Straight Line & 51 & $100 \%$ & 1 & \\
\hline a) Used & 34 & $67 \%$ & 0,6667 & \multirow{2}{*}{0,556} \\
\hline b) Not used/Not mentioned & 17 & $33 \%$ & 0,3333 & \\
\hline Degressive & 51 & $100 \%$ & 1 & \\
\hline a) Used & 0 & $0 \%$ & 0 & \multirow{2}{*}{1} \\
\hline b) Not used/Not mentioned & 51 & $100 \%$ & 1 & \\
\hline Accelerated & 51 & $100 \%$ & 1 & \\
\hline a) Used & 1 & $2 \%$ & 0,0196 & \multirow{2}{*}{0,962} \\
\hline b) Not used/Not mentioned & 50 & $98 \%$ & 0,9804 & \\
\hline Production Units & 51 & $100 \%$ & 1 & \\
\hline a) Used & 0 & $0 \%$ & 0 & \multirow{2}{*}{1} \\
\hline b) Not used/Not mentioned & 51 & $100 \%$ & 1 & \\
\hline
\end{tabular}

The last case for impairment consists in customers' lists, whose analysis generates a medium to high accounting harmonization (table no.14). 


\section{Impairment for Customers Lists}

Table no. 14

\begin{tabular}{ccccc}
$\begin{array}{c}\text { Treatment/ } \\
\text { Accounting } \\
\text { option }\end{array}$ & $\begin{array}{c}\text { Companies } \\
\text { No. }\end{array}$ & Frequency & pi & $\begin{array}{c}\text { H } \\
\text { Index }\end{array}$ \\
\hline
\end{tabular}

\begin{tabular}{ccccc}
\hline IMPAIRMENT & & & & \\
\hline Determination Models & 51 & $100 \%$ & 1 & \\
\hline Impariment testing & 5 & $10 \%$ & 0,0980 & 0,823 \\
\hline a) Used & 46 & $90 \%$ & 0,9020 & \\
b) Not used/Not mentioned & 51 & $100 \%$ & 1 & \\
\hline Amortization & 30 & $59 \%$ & 0,5882 & 0,516 \\
\hline a) Used & 21 & $41 \%$ & 0,4118 & \\
b) Not used/Not mentioned & & & & \\
\hline Amortization Methods & 51 & $100 \%$ & 1 & \\
\hline Linear/Straight Line & 25 & $49 \%$ & 0,4902 & 0,500 \\
\hline a) Used & 26 & $51 \%$ & 0,5098 & \\
b) Not used/Not mentioned & 51 & $100 \%$ & 1 & \\
\hline Degressive & 0 & $0 \%$ & 0 & 1 \\
\hline a) Used & 51 & $100 \%$ & 1 & \\
b) Not used/Not mentioned & 51 & $100 \%$ & 1 & \\
\hline Accelerated & 1 & $2 \%$ & 0,0196 & \multirow{2}{*}{0,962} \\
\hline a) Used & 50 & $98 \%$ & 0,9804 & \\
b) Not used/Not mentioned & 51 & $100 \%$ & 1 & \\
\hline Production Units & 0 & $0 \%$ & 0 & \\
\hline a) Used & 51 & $100 \%$ & 1 & \\
b) Not used/Not mentioned & & & & \\
\hline
\end{tabular}

The last part of the research implies aggregating the values obtained for $\mathrm{H}$ Index. This part of the analysis assumes computing the average for each of the intangibles, as well as for each of the accounting method (table no.15). The findings of the research are synthesised in the following table, in which the second column represents averages for Indexes calculated and presented previously, while the last or third column consists in general averages for the assets stages, namely recognition, valuation and impairment. Regarding valuation, averages were also computed for initial valuation and revaluation, and afterwards a general average for valuation was calculated. The same applies to determination models and amortization methods in case of impairment. Finally, the main average was obtained, by considering the three stages mentioned before. The final result indicates a high degree for harmonization, $H$ Index recording a value of 0,7 . Therefore, the research findings provide evidence for the initial hypothesis that IFRS has indeed generated accounting practices harmonization within European Union member states. 


\section{Aggregated Values for $\mathrm{H}$ Index}

Table no. 15

\begin{tabular}{|c|c|c|}
\hline \multicolumn{3}{|c|}{ Aggregated Values for $H$ Index } \\
\hline $\begin{array}{c}\text { Treatment/ } \\
\text { Accounting option }\end{array}$ & H Index & H Index \\
\hline \multicolumn{3}{|l|}{ RECOGNITION } \\
\hline Set up costs & 0,664 & \multirow{6}{*}{0,796} \\
\hline Development costs & 0,640 & \\
\hline Goodwill & 1 & \\
\hline Trademarks/Brands & 1 & \\
\hline Patents/Licences & 1 & \\
\hline Customer Lists & 0,608 & \\
\hline \multicolumn{3}{|l|}{ VALUATION } \\
\hline \multicolumn{3}{|l|}{ Initial Valuation } \\
\hline Acquisition cost & 0,576 & \multirow{2}{*}{0,592} \\
\hline Production cost & 0,608 & \\
\hline \multicolumn{3}{|l|}{ Revaluation } \\
\hline Book Value & 0,572 & \multirow{2}{*}{0,601} \\
\hline \multirow[t]{2}{*}{ Fair Value } & 0,630 & \\
\hline & & 0,597 \\
\hline \multicolumn{3}{|l|}{ IMPAIRMENT } \\
\hline \multicolumn{3}{|l|}{ Determination Models } \\
\hline Impariment testing & 1 & \multirow{2}{*}{0,723} \\
\hline Amortization & 0,645 & \\
\hline \multicolumn{3}{|l|}{ Amortization Methods } \\
\hline Linear/Straight Line & 0,645 & \multirow{4}{*}{0,729} \\
\hline Degressive & 1 & \\
\hline Accelerated & 1 & \\
\hline \multirow[t]{3}{*}{ Production Units } & 0,981 & \\
\hline & & 0,726 \\
\hline & & 0,706 \\
\hline
\end{tabular}

\section{Conclusions}

The aim of this paper was to study the impact of IFRS on accounting practices harmonization. This has been achieved by measuring the degree in which different companies from Europe use same methods when reporting their intangible assets. The initial hypothesis stated that IFRS has generated accounting practices harmonization within European Union member states. In an attempt to demonstrate the existence of harmonizing tendencies between European countries, as a result of IFRS adoption, we used a specific methodology that implies Herfindhal Index computation for a sample of 51 listed companies. In addition, these firms are known to develop their activity in five European Union member states. According to initial estimations, it has been assumed that International Financial Reporting Standards had a certain influence on accounting practices, which in turn would results in evidence of high harmonization level for intangible assets. 
On the basis of both theoretical background and quantitative research, we can underline the tendency of harmonization processes in what concerns accounting practice of the analysed companies.

When measuring the comparability degree of accounting practices corresponding to the companies from the analysed sample, we determined $\mathrm{H}$ Index for the accounting treatments and options, and finally we computed an average of these indicators. Further on, the obtain results suggest the following: a high level of harmonization for recognition of intangibles, a tendency to reconciliation for valuation of these assets, as well as common practices concerning impairment.

The findings suggest the influence of International Financial Reporting Standards on accounting practices, as there is prove of high harmonization level for intangible assets. Further on, they provide evidence for the initial hypothesis that IFRS has generated accounting practices harmonization within European Union member states. Also, the fact that most of the analysed situations recorded if not maximum harmonizing values at least visible tendencies to harmonize accounting practices, represents another element that sustains the initial hypothesis.

Regarding the paper contribution to international accounting, measuring material harmonization for companies activating in Europe and analysing their accounting policies in order to establishing the harmonization degree for the particular case of intangible assets, concurs to the development of accounting field on both national and international levels.

\section{References}

1. Canibano, L., Mora, A., 2000. Evaluating the statistical significance of de facto accounting harmonization: a study of European global players. European Accounting Review 9.

2. Capalbo, F., 2003. Domestic Practice, Comparative International Analysis and Accounting Discretion- Italian Savings Banks' Pensions Cost Accounting. Abacus 39.

3. Cazavan, J., A., Stolowy, H., 2001. International accounting disharmony: the case of intangibles. Accounting, Auditing and Accountability Journal 14.

4. Chand, P., White, M., 2007. A critique of the influence of globalization and convergence of accounting standards in Fiji. Critical Perspectives on Accounting 18.

5. Combarros, J., L., L., 2000. Accounting and financial audit harmonization in the European Union. European Accounting Review 9.

6. Ding, Y., Hope, K., O., Jeanjean T., Stolowy, H., 2007. Differences between domestic accounting standards and IAS: Measurement, determinants and implications. Journal of Accounting and Public Policy 26.

7. Fuertes, I., 2008. Towards Harmonization or Standardization in Governmental Accounting? The International Public Sector Accounting Standards Board Experience. Journal of Comparative policy Analysis 10.

8. Hancock, P., Taplin, R., Tower, G., 2002. Disclosure and compliance of accounting policies: Asia-Pacific evidence. Accounting Forum 26.

9. Kikuya, M., 2001. International harmonization of Japanese accounting standards. Accounting, Business and Financial History 11.

10. Lin, J., Z., Wang, L., 2001. Financial disclosure and accounting harmonization: cases of three listed companies in China. Managerial Auditing Journal 16.

11. Muhammad, J., A., 2006. Disclosure harmonization of accounting practices: the case for South Asia. Asian Review of Accounting 14.

12. Ramcharran, H., 2000. The need for International Accounting Harmonization: An examination and Comparison of the Practices of Russian Banks. American Business Review 18.

13. Reinstein, A., Weirich, R., T., 2002. US and UK GAAP: Important Differences for Financial Statements Preparers and Users. Managerial Finance 28. 
14. Taplin, R., H., 2003. Harmony, Statistical Inference with the Herfindahl H Index and C Index. Abacus 39.

15. Taplin, R., H., 2004. A unified approach to the measurement of international accounting harmony. Accounting and Business Research 34.

16. www.boerse-frankfurt.com

17. www.borsaitaliana.com

18. www.deutsche-boerse.com

19. www.euronext.com

20. www.londonstockexchange.com. 